University of Nebraska - Lincoln

DigitalCommons@University of Nebraska - Lincoln

Phylogenomic and functional domain analysis of polyketide synthases in Fusarium

\author{
Daren W. Brown \\ USDA-ARS, daren.brown@ars.usda.gov \\ Robert A. E. Butchko \\ USDA-ARS \\ Scott E. Baker \\ Pacific Northwest National Laboratory \\ Robert A. Proctor \\ USDA-ARS
}

Follow this and additional works at: https://digitalcommons.unl.edu/usdaarsfacpub

Brown, Daren W.; Butchko, Robert A. E.; Baker, Scott E.; and Proctor, Robert A., "Phylogenomic and functional domain analysis of polyketide synthases in Fusarium" (2012). Publications from USDA-ARS / UNL Faculty. 1583.

https://digitalcommons.unl.edu/usdaarsfacpub/1583

This Article is brought to you for free and open access by the U.S. Department of Agriculture: Agricultural Research Service, Lincoln, Nebraska at DigitalCommons@University of Nebraska - Lincoln. It has been accepted for inclusion in Publications from USDA-ARS / UNL Faculty by an authorized administrator of DigitalCommons@University of Nebraska - Lincoln. 


\title{
Phylogenomic and functional domain analysis of polyketide synthases in Fusarium
}

\author{
Daren W. BROWN ${ }^{a, *}$, Robert A. E. BUTCHKO ${ }^{a}$, Scott E. BAKER ${ }^{b}$, Robert H. PROCTOR \\ ${ }^{a}$ Bacterial Foodborne Pathogens and Mycology Research, USDA-ARS-NCAUR, 1815 N. University St., Peoria, Illinois 61604, USA \\ ${ }^{\mathrm{b} C h e m i c a l}$ and Biological Process Development Group, Pacific Northwest National Laboratory, Richland, Washington 99352, USA
}

\section{A R T I C L E I N F O}

\section{Article history:}

Received 13 September 2011

Received in revised form

9 December 2011

Accepted 10 December 2011

Available online 19 December 2011

Corresponding Editor:

Kerry O'Donnell

\section{Keywords:}

Fusarium

Microarray

Phylogenomic analysis

Polyketide

Secondary metabolites

\begin{abstract}
A B S T R A C T
Fusarium species are ubiquitous in nature, cause a range of plant diseases, and produce a variety of chemicals often referred to as secondary metabolites. Although some fungal secondary metabolites affect plant growth or protect plants from other fungi and bacteria, their presence in grain-based food and feed is more often associated with a variety of diseases in plants and in animals. Many of these structurally diverse metabolites are derived from a family of related enzymes called polyketide synthases (PKSs). A search of genomic sequence of Fusarium verticillioides, Fusarium graminearum, Fusarium oxysporum, and Fusarium solani identified a total of 58 PKS genes. To gain insight into how this gene family evolved and to guide future studies, we conducted phylogenomic and functional domain analyses. The resulting geneaology suggested that Fusarium PKSs represent 34 different groups responsible for synthesis of different core metabolites. The analyses indicate that variation in the Fusarium PKS gene family is due to gene duplication and loss events as well as enzyme gain-of-function due to the acquisition of new domains or of loss-offunction due to nucleotide mutations. Transcriptional analysis indicates that the $16 \mathrm{~F}$. verticillioides PKS genes are expressed under a range of conditions, further evidence that they are functional genes that confer the ability to produce secondary metabolites.
\end{abstract}

Published by Elsevier Ltd on behalf of The British Mycological Society.

\section{Introduction}

A hallmark of fungi is their capacity to synthesize a wide variety of structurally diverse natural products (Fig 1) (Cole et al. 2003). The frequently associated biological activity of these metabolites has positively impacted the pharmaceutical industry (e.g. the cholesterol-lowering drug lovastatin and the antibiotic cephalosporin) and negatively impacted the agricultural industry (e.g. the mycotoxins fumonisin, zearalenone, and trichothecene). Natural products that are not required for life or for propagation are often called secondary metabolites (SMs) (Bennett \& Bentley 1989; Keller et al. 2005). Consumption of mycotoxin-contaminated grain or grain-based food and feed has been associated with a variety of human and animal diseases. Economic losses caused by mycotoxin contamination of grain can be in the 100s of millions of dollars each year due to negative impacts on human and animal health and reduced value of grain (Glenn 2007; Morgavi \& Riley 2007; Wu 2007). Ingesting contaminated food may have an immediate (toxic) or delayed impact. For example, fumonisin can cause leukoencephalomalacia in horses and has been epidemiologically associated with neural tube defects in newborns and esophageal cancer in adults (Sydenham et al. 1990.; Seefelder et al. 2003; Marasas et al. 2004; Voss et al. 2007).

Many fungal SMs are polyketides, including the mycotoxins fumonisins and zearalenone. Polyketides are typically

\footnotetext{
* Corresponding author. Tel.: +1 309681 6230; fax: +1 3096816672.

E-mail address: daren.brown@ars.usda.gov

1878-6146/\$ - see front matter Published by Elsevier Ltd on behalf of The British Mycological Society.

doi:10.1016/j.funbio.2011.12.005
} 
<smiles>CCCCC(C(=O)O)C1C(=O)OC(CC(C)CC(O)CCCCC(O)CC(O)C(C)N)C(OC(=O)CC(CC(=O)O)C(=O)O)C1CCCC</smiles><smiles>COc1cc(C)c2c(=O)c3c(O)c4c(c(O)c3oc2c1)C(=O)C(OC)CC4=O</smiles>

Bikaverin: NR-PKS: FvFo<smiles>COc1cc(O)c2c(c1O)C(=O)C1=C(COC(C)(O)C1)C2=O</smiles>

Fusarubin: NR-PKS: FvFvFgNh<smiles>COC1=C(C2=C(OC)C(=O)c3cc4oc(C)cc(=O)c4c(O)c3C2=O)C(=O)c2c(cc3oc(C)cc(=O)c3c2O)C1=O</smiles>

Aurofusarin: NR-PKS: Fg<smiles>CO[C@H](C)CCCC(=O)CCC/C=C/c1cc(O)cc(O)c1C(=O)O</smiles>

Zearalenone: NR-PKS and R-PKS: Fg<smiles>CCCCc1ccc(C(=O)O)nc1</smiles>

Fusaric acid: R-PKS: FvFo<smiles>[R20]SP=O</smiles>

Fusarielin A: R-PKS: Fg<smiles>CC1(C)S[C@@]2(C)[C@H](NC(=O)Cc3ccccc3)C(=O)N2[C@H]1C(=O)O</smiles>

Penicillin G: NRPS: Pen

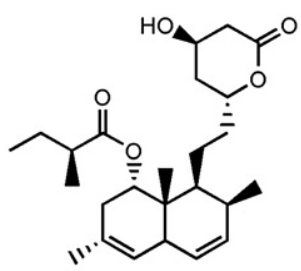

Lovastatin: R-PKS: Asp

Fig 1 - Diverse chemical structures of fungal polyketide-derived metabolites. The type of core enzyme involved in metabolite synthesis and the fungi associated with their synthesis are indicated. Fv = F. verticillioides; Fg = F. graminearum; Fo = F. oxysporum; Nh = F. solani; Asp = Aspergillus; Pen = Penicillium. NR-PKS-NRPS $=$ NR-PKS with an NRPS module. The metabolites are made by the Fusarium species indicated while the two metabolites in the inset are examples of fungal derived pharmaceutical.

synthesized by polyketide synthases (PKSs) from small carboxylic acid derivatives (acetyl-CoA and malonyl-CoA) in a manner similar to the synthesis of fatty acids by a fatty acid synthase (FAS) (Hopwood \& Khosla 1992). Fungal PKSs contain five to eight functional domains that are fused into one large protein and are used iteratively (Fig 2). PKSs can be divided into two major groups based on their domain content: non-reducing PKSs (NR-PKSs) synthesize polyketides in which carbonyl groups are not reduced, and reducing PKSs (R-PKS) synthesize polyketides in which the carbonyl groups are partially or fully reduced. Polyketide synthesis is initiated by the transfer of a starter unit (typically acetyl-CoA) onto the phosphopantetheine group bound to the acylcarrier protein (ACP) domain of the PKS by the acyltransferase (AT) domain of R-PKSs and by the starter unit AT (SAT) domain of NR-PKSs. Next, the ketoacyl synthase (KS) domain catalyzes transfer of the acetyl unit from the ACP to the KS and decarboxylative condensation with an elongation unit (typically malonyl-CoA). Prior to the condensation, an elongation unit is loaded onto the ACP domain by the AT domain of R-PKSs or the malonyltransferase domain (MAT) of NRPKSs. R-PKSs generally remove the carbonyl group (i.e. $\beta$-keto) of the resulting four-carbon molecule via a succession of reactions: the keto reductase (KR) domain catalyzes reduction of the $\beta$-keto to a hydroxyl; the dehydratase (DH) domain catalyzes reduction of the hydroxyl to an enoyl (carbon-carbon double bond); and the enoyl reductase (ER) domain catalyzes reduction of the enoyl to an alkane (carbon-carbon single bond). Because they generally lack $\mathrm{KR}, \mathrm{DH}$, and ER domains, NR-PKSs usually do not reduce the $\beta$-keto group. The cycle is initiated a second time with the condensation of the four-carbon intermediate with another malonyl-CoA. The total number of cycles (generally between two and nine), product cyclization, and release of the polyketide from the PKS are mediated by the KS, the product template (PT), and terminal thioesterase (TE) or reductase (R) domains (Crawford \& Townsend 2010; Du \& Lou 2010). The presence of carbonyl groups along the carbon backbone of NR-PKSs generally lead to metabolites with one or more aromatic rings, whereas R-PKSs typically give rise to metabolites with a reduced carbon chain. Additional, structural diversity of polyketides can arise from three other sources: (1) incomplete reduction of one or more $\beta$-keto groups, (2) additional modifying domains [e.g. methyltransferase (ME) domain or non-ribosomal peptide synthase module (NRPS)] in the PKS, and (3) other enzymes that catalyze structural modifications (e.g. oxygenations, reductions, acylations, or isomerizations) following synthesis of the polyketide. 


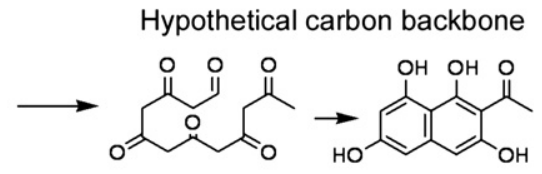

B Reducing PKS (2200 to 4000 AAs)

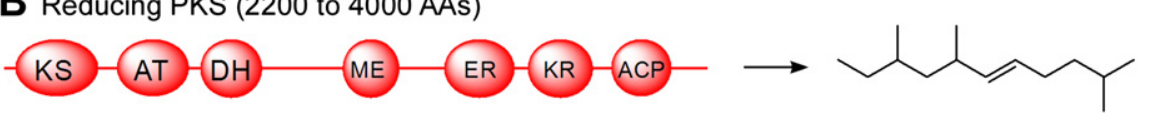

Fig 2 - Generalized domain organization of fungal NR-PKSs and R-PKSs. (A) NR-PKS range in size from 2000 to 2300 AAs. All Fusarium NR-PKSs have five invariable domains (SAT, KS = ketoacyl synthase, MAT, PT domain and ACP). Some NR-PKSs may have a second ACP domain. In NR-PKSs, the carboxy-terminal domain is typically a TE or R. One predicted NR-PKS (FGSG_04588) has a terminal ME. (B) R-PKS range in size from 2200 to 4000 AAs. All Fusarium R-PKSs have five invariable domains (KS, AT, DH, KR, and ACP). Two domains, ME and ER, may or may not be functional. Of the 47 total R-PKSs, 31 have an ME domain located after the DH domain while 36 have an ER domain just prior to the KR domain. Remnant ME and ER domains are present in the remaining R-PKSs respectively. Eight (17\%) R-PKSs include a NRPS domain and four (8.5 \%) include a CAT (carnitine AT domain).

Studies examining Fusarium focus primarily on their plant pathogenic nature and their ability to contaminate grains and grain products with mycotoxins (Fig 1). Fusarium is a diverse group of fungi, and, in addition to causing significant crop disease, may live unnoticed in plants and soils as endophytes and saprophytes. The name Fusarium refers to the asexual phase (anamorph) of these fungi. Their sexual phase (teleomorph) is Gibberella. Nectria haematococca (synonym Haematonectria haematococca) is the teleomorphic name for the anamorph Fusarium solani, so named because like fusaria with a Gibberella teleomorph it produces multi-celled, spindle-shaped asexual spores (macroconidia). Nevertheless, fusaria with Gibberella and Nectria teleomorphs represent two phylogenetically distinct lineages within the fungal family Nectriaceae (order Hypocreales, phylum Ascomycota) (O’Donnell et al. 2008).

A recent comparative genomic study of Fusarium graminearum, Fusarium oxysporum, and Fusarium verticillioides identified 46 potential secondary metabolite biosynthesis gene clusters, of which $87 \%$ include a PKS gene (Ma et al. 2010). The number of polyketide-derived metabolites $(\geq 15)$ potentially synthesized by any one of these species is significantly greater than the number of metabolites currently attributed to them [reviewed in (Desjardins \& Proctor 2007)]. For example, to date, the function of five of the $16 \mathrm{~F}$. verticillioides PKSs are known. FUM1 is involved in fumonisin synthesis (Proctor et al. 1999), FUS1 is involved in fusarin synthesis (Song et al.
2004), PGL1 is involved in the synthesis of a violet perithecial pigment (Proctor et al. 2007), BIK1 is involved in bikaverin synthesis (Brown et al. 2008) and FAB1 is involved in fusaric acid synthesis (Brown et al. submitted for publication). Phylogenetic analysis resolved the 58 Fusarium PKSs into four major clades and revealed a high degree of diversity with few closely related orthologues (Ma et al. 2010), results consistent with previous studies examining PKSs from multiple, diverse genera of the Ascomycota (Kroken et al. 2003; Baker et al. 2006).

In this study, we used previously reported (Ma et al. 2010) phylogenetic analysis of 58 Fusarium PKSs to: (1) examine the content of functional domains in the PKSs and (2) identify species-specific orthologues and predict chemical products of PKSs based on their relatedness to PKSs for which the corresponding SM has been identified. The identification of species-specific PKSs and orthologous groups of PKSs in two, three or all four Fusarium will help direct further studies aimed at characteristics of PKSs that are unique to one or a few or common to many Fusarium species (Table 1).

\section{Materials and methods}

Search for PKSs

Fusarium verticillioides, Fusarium graminearum, and Fusarium oxysporum PKS genes were identified by Basic Local Alignment

Table 1 - Types of Fusarium PKSs.

\begin{tabular}{|c|c|c|c|c|c|}
\hline Species & Total PKSs & NR-PKSs & R-PKSs & R-PKS-NRPS & R-PKS-CAT \\
\hline F. verticillioides & 16 & 2 & 11 & 3 & - \\
\hline F. oxysporum & 14 & 2 & 8 & 3 & $1^{\mathrm{a}}$ \\
\hline F. graminearum & 15 & 5 & 8 & 1 & 1 \\
\hline \multirow[t]{2}{*}{ F. solani } & 13 & 2 & 8 & 1 & 2 \\
\hline & 58 & & & & \\
\hline
\end{tabular}


Search Tool (BLAST) analysis against all predicted proteins (Altschul et al. 1990) at the Broad Institute (http://www.broad institute.org/annotation/genome/fusarium_verticillioides/ MultiHome.html), and Fusarium solani PKS genes were identified at the Joint Genome Institute (JGI) (http://genome.jgipsf.org/) websites as described (Ma et al. 2010).

\section{Phylogenetic analysis}

The phylogram representing the phylogenetic relationships between Fusarium PKSs (Fig 3) was constructed based on an alignment of amino acid (AA) sequences of the $\mathrm{KS}$ and AT/MAT domains from all 58 PKSs as described (Ma et al. 2010). AA sequence alignments were done with the ClustalW+ program in the Genetics Computer Group (GCG) analysis software package Version 11.1.3-UNIX, Accelrys Inc., San Diego, CA, USA (Devereux et al. 1984) using the Blosum multiple sequence alignment scoring matrix. Phylogenetic analyses were done using the GCGs PaupSearch and PAUP* version 4.0b10-Unix [Phylogenetic Analysis Using Parsimony (PAUP)]. Statistical support for branches within the phylogenetic trees was generated by bootstrap analysis with 1000 pseudoreplicates. Two-AA sequence comparisons were carried out using the DNAMAN software (Lynnon BioSoft, Vaudreuil, Quebec, Canada).

\section{Expression analysis}

Gene expression in wild-type Fusarium verticillioides strain FRC M-3125 was analyzed by Expressed Sequence Tags (ESTs) and by microarray under different developmental, nutritional, and pathogenic conditions. ESTs (transcripts) corresponding to the F. verticillioides PKS genes were retrieved from the F. verticillioides Gene Index database at the DanaFarber Cancer Institute (http://compbio.dfci.harvard.edu/tgi/ cgi-bin/tgi/gimain.pl?gudb=f_verticill). The Gene Index is composed of 13350 sequences derived from over 86908 ESTs collected from ten $F$. verticillioides cDNA libraries (Brown et al. 2005). Libraries FvF, FvG, FvM, and FvO were generated from cultures grown on GYAM $(0.24 \mathrm{M}$ glucose, $0.05 \%$ yeast extract, $8 \mathrm{mM}$ L-asparagine, $5 \mathrm{mM}$ malic acid, $1.7 \mathrm{mM}$ $\mathrm{NaCl}, 4.4 \mathrm{mM} \mathrm{K}_{2} \mathrm{HPO}_{4}, 2 \mathrm{mM} \mathrm{MgSO}_{4}$, and $8.8 \mathrm{mM} \mathrm{CaCl}_{2}, \mathrm{pH}$ 3.0) for $24 \mathrm{~h}$ (FvF), 48/72 h (FvM), $96 \mathrm{~h}$ (FvG) from M-3125 and $90 \mathrm{~h}(\mathrm{FvO})$ with the $\mathrm{F}$. verticillioides fumonisin non-producing strain 57-7-7 (Desjardins et al. 1996). Library FvN was generated from M-3125 grown on corn meal medium for 4 and 6 d, library FvI from M-3125 grown on excised maize seedling root and shoots and library FvH from germinating spores of $\mathrm{M}-3125$ after $10 \mathrm{~h}$ in water extracts of maize seedlings (Brown et al. 2005).

Microarrays were designed and produced by Roche NimbleGen (Madison, WI, USA). Each sequence is represented on the array by between six and 12 unique 60 -mer probes. Total RNA was extracted using the RNeasy Mini kit (Qiagen Inc., Valencia, CA, USA). Microarray hybridization, data acquisition, and initial analysis were conducted by Roche NimbleGen, Iceland. Data were normalized by robust multi-array average (Bolstad et al. 2003; Irizarry et al. 2003) and were compared using the Acuity 4.0 microarray analysis software package (Molecular Devices Corp, Sunnyvale, USA). A brief description of the ten different microarray experiments referred to in Fig 8 are as follows. In general, samples for RNA extraction were collected after growth on different substrates for different intervals of time, flash frozen by immersion in liquid nitrogen and stored at $-80^{\circ} \mathrm{C}$ prior to RNA extraction The first two experiments were conducted on liquid media with shaking. In the GYAM experiment, samples were collected after 24, 48, 72, and $96 \mathrm{~h}$ of growth (Gene Expression Omnibus (GEO) accession no. GSE16900 (Brown 2011)). In the potato dextrose broth [( $\underline{\mathrm{PDB}})$, Difco, Detroit, MI, USA] experiment, samples were collected after 24 and $48 \mathrm{~h}$ of growth. The next two experiments were conducted on autoclaved maize kernels. In the En/Germ experiment, samples were collected after growth on either excised kernel endosperm or germ tissue (Shim et al. 2003). In the CC 48 experiment, samples were collected after growth on cracked corn for $48 \mathrm{~h}$. The next three experiments were conducted on live maize ears. In the Ear 3-7 experiment, infected kernels were collected 3 and $7 \mathrm{~d}$ after inoculation. In the Ear Symptomatic experiment, infected kernels displaying significant disease symptoms were collected $21 \mathrm{~d}$ after inoculation. In the Live Seeds experiment, live maize seeds were inoculated and collected after 4 and $8 \mathrm{~d}$. The next three experiments were conducted after growth in maize seedlings roots. In the SRIVW experiment, infected maize seedling roots were collected after 2, and 4 weeks of growth; in the SRIIIW experiment, infected maize seedling roots were collected after 5,8 , 14 , and $21 \mathrm{~d}$ of growth; and in SRIIIWS experiment, infected maize seedling roots were also collected after $5,8,14$, and $21 \mathrm{~d}$ of growth except that in this case, the seedlings were not watered between day 8 and day 13. PKS genes were assigned as 'expressed' if the expression level changed $>2$-fold over the course of one experiment or if expression changed $>4$-fold in one experiment relative to another.

\section{Results and discussion}

\section{PKS genealogy}

Phylogenomic analysis of PKSs can delineate distinct orthologous groups of PKSs shared by one or more species. Such delineation can provide insight into characteristics of PKSs that affect metabolite production and into biosynthetic potential of species. Previously, we surveyed the four Fusarium genome sequence databases and identified 58 PKS genes (Table 1) (Ma et al. 2010). Maximum parsimony analysis of alignments of the AA sequences of the combined KS and AT/ MAT domains resolved Fusarium PKSs into two major clades that correspond to NR-PKSs and R-PKSs based on functional domain content (Fig 3) (Ma et al. 2010). The KS-AT/MAT genealogy reported here is consistent with previously reported KS genealogies from multiple fungal genera (Kroken et al. 2003; Baker et al. 2006). The resolution of NR-PKSs into a single clade in the KS-AT/MAT tree supports the hypothesis that Fusarium NR-PKSs are monophyletic; i.e. they share a common ancestor (Fig 3). However, based on previous analyses with multiple fungal genera (Kroken et al. 2003; Baker et al. 2006), the putative ancestral NR-PKS existed before divergence of Fusarium from other fungi. In the Fusarium genealogy, the R-PKSs were resolved into three clades with significant bootstrap support 


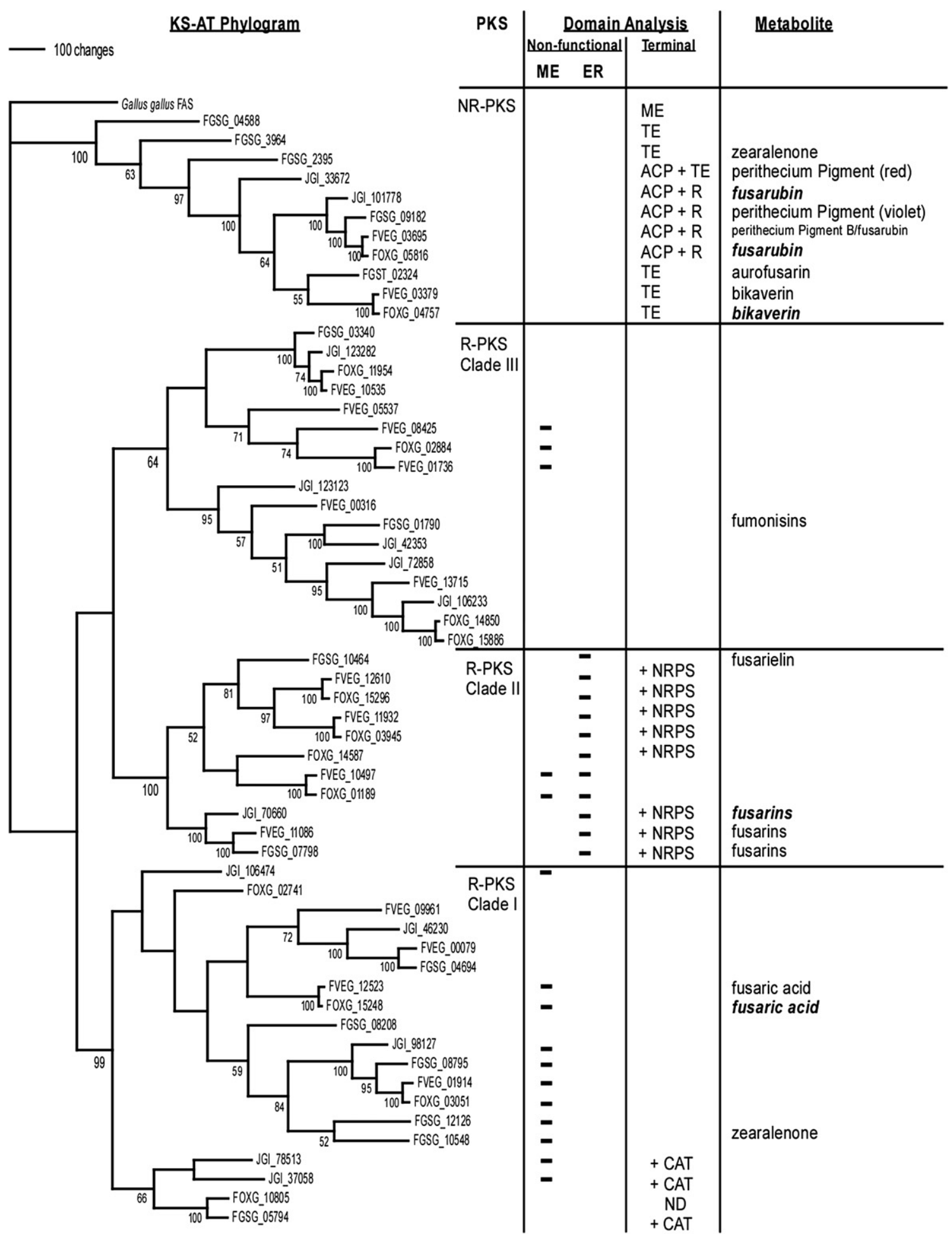

Fig 3 - Genealogy of the Fusarium PKSs inferred by maximum parsimony analysis of deduced AA sequences of the KS and AT/MAT domains. Major clades are demarked by horizontal lines to the right of the tree. Numbers below branches indicate percentage bootstrap support for each branch point based on 1000 pseudoreplicates. The FAS from Gallus gallus served as an outgroup. Designations beginning with FGSG, FOXG, and FVEG correspond to the F. graminearum, F. oxysporum, and F. verticillioides, respectively, gene/protein models from the Broad Institute's Fusarium Comparative Database. Designations beginning with JGI correspond to F. solani gene/protein models from JGI's genome sequence database. The dashes in the Mutant Domain column under ME and ER heading refer to the presence of a non-functional ME or ER domain, respectively, in the predicted PKSs. The carboxy-terminal domains (TE, R, and ACP) are indicated in the Terminal column for the NR-PKS clade. The presence of an additional NRPS or CAT (carnitine acyltransferase) domain in R-PKSs is indicated by a plus sign. When known, the secondary metabolites corresponding to a PKS are listed. Metabolite names that are italicized and in bold text are predicted products. 
(Fig 3) (Ma et al. 2010). Based on the conserved organization of multiple functional domains, as well as previous phylogenetic analyses (Kroken et al. 2003), it is likely that Fusarium R-PKSs also evolved from a common ancestor.

\section{Orthologous PKSs}

The phylogenetic analysis of KS-AT/MAT domains also indicated the presence of closely related PKS orthologues in two or more of the species examined (Figs 3 and 4). For example, the set of four closely related PKSs (with $100 \%$ bootstrap support and identical functional domains) FGSG_09182, FVEG_03695, FOXG_05816, and JGI_101778 includes two required for synthesis of the violet perithecial pigment in Fusarium verticillioides and Fusarium graminearum (Gaffoor et al. 2005; Proctor et al. 2007) and fusarubin in F. verticillioides

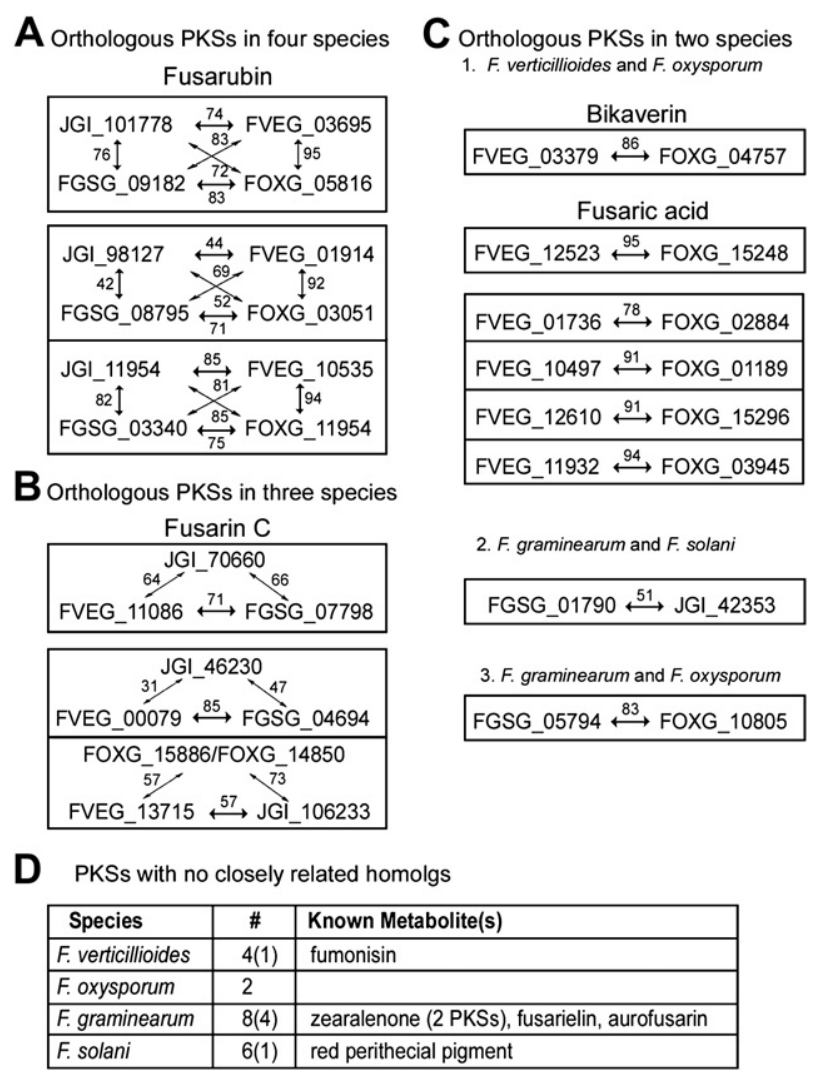

Fig 4 - Groups of orthologous PKSs that share $100 \%$ bootstrap support. Numbers above arrows represent percent AA identity shared between predicted PKSs. When metabolite corresponding to PKS/group is known, the metabolite name is indicated immediately above each group. (A) Orthologous sets of PKSs present in all four Fusarium species. (B) Orthologous sets of PKSs present in three Fusarium. Since FOXG_15886 and FOXG_14850 share $97 \%$ AA identity and were only present in $F$. oxysporum, they were considered to be paralogous. (C) Orthologous PKSs present in two Fusarium species. (D) Number of unique PKSs in each of the four Fusarium species and the number of PKSs with known function, in parentheses. Two different PKSs are involved in zearalenone synthesis by F. graminearum.
(Busman and Proctor, unpublished observations). Likewise, the set of three orthologues (FGSG_07798, FVEG_11086, and JGI_70660) include two required for fusarin production in F. verticillioides and F. graminearum (Gaffoor et al. 2005; Brown et al. submitted for publication). Although the closely related PKS orthologues are expected to synthesize the same polyketide, the structure and, by extension, ecological function of the $\mathrm{SM}$ end-product may differ in each fungus. For example, the putative gene cluster associated with the PKS gene required for the violet perithecial pigment consists of the same six genes in F. graminearum, Fusarium oxysporum, and F. verticillioides (Gaffoor et al. 2005; Proctor et al. 2007; Brown et al. submitted for publication). However, the putative cluster in Fusarium solani includes the PKS gene (JGI_101778) and only two of the other five genes present in the Fusarium species (Proctor et al. 2007). Thus, any putative product of the putative F. solani cluster is likely to be different than that of the clusters in the Fusarium species. In addition, a red pigment rather than a violet one accumulates in the perithecial walls of F. solani, and a different PKS (JGI_33672) is required for its synthesis (Graziani et al. 2004).

We identified three PKS genes with closely related orthologues in three of four species examined. The function of only one of these PKSs has been determined; the F. graminearum gene FGSG_07798 and its F. verticillioides orthologue (FVEG_11086) are required for production of fusarin mycotoxins (Song et al. 2004; Gaffoor et al. 2005; Brown et al. submitted for publication). Again, the genes flanking the fusarin PKS in F. graminearum and F. verticillioides (Brown et al. submitted for publication) do not exactly match the sets of genes flanking the F. solani orthologue JGI_70660, suggesting that once the polyketide is synthesized it undergoes different chemical modifications in F. solani versus F. verticillioides and F. graminearum. Because of the high degree of similarity shared by FOXG_15886 and FOXG_14850 (97 \% identity) and the $100 \%$ bootstrap support shared between this set and FVEG_13715 and JGI_106233, these four PKSs are considered an orthologous set of three PKSs (i.e. a pair from F. oxysporum and one each from $F$. verticillioides and $F$. solani).

We also identified eight PKS orthologues that occur only in two of the species examined. Six of these orthologous PKSs occur in F. verticillioides and F. oxysporum, which are the most closely related species included in this analysis (Ma et al. 2010). Overall, 12 of the PKSs in these two fungi are closely related orthologues, implying that most of the polyketides produced by these species are structurally very similar. In contrast, almost half of the PKSs from F. graminearum and F. solani are unique to each fungus (Fig 4D). The last two orthologous pairs are shared by F. oxysporum and F. graminearum and F. oxysporum and F. solani (Fig 4C) and presumably reflects a shared function unique to these fungi. In contrast, the lack of a shared pair of orthologous PKSs by F. verticillioides and F. graminearum, both pathogens of maize, suggests that a common PKS product may not be critical for pathogenesis on maize.

The phylogenetic analysis has resolved Fusarium PKSs into 14 clades of two (8), three (3), and four (3) likely orthologous PKSs (adjacent PKSs with branch points of $100 \%$ bootstrap support) (Figs 3 and $4 \mathrm{~A}-\mathrm{C}$ ) and 20 PKSs that lack closely related orthologues among the species examined (Fig 4D). 
Thus, the analysis suggests that there are 34 distinct groups of PKSs among the four species. Percent identity between putative orthologues ranges from $31 \%$ to $94 \%$. In some cases, orthologous PKSs required for synthesis of the same metabolites have been characterized in multiple Fusarium species (e.g. fusarin PKS; $64-71 \%$ ). Based on the percent identity of these characterized putative orthologues, it is likely that the 34 groups of PKSs are responsible for synthesis of at least 34 structurally distinct groups of polyketides, only seven of which would be produced by both Fusarium and Nectria.

\section{Gene duplication}

Two Fusarium oxysporum PKSs, FOXG_14850, and FOXG_15886, appear to have arisen by a gene duplication event. The PKS coding sequences, as well as flanking sequence $(2.5 \mathrm{~kb}$ upstream and $3.2 \mathrm{~kb}$ downstream), share $98 \%$ identity for a total duplicated genomic segment of $14.5 \mathrm{~kb}$. The entire segment may represent a three-gene cluster as the upstream and downstream sequences are each predicted to encode a different methyltransferase (FOXG_14851 and FOXG_15882 downstream and FOXG_14849 and FOXG_15887 upstream from each PKS respectively), enzymes that are often involved in secondary metabolism. A 767-bp insertion just upstream of the predicted ATG of FOXG_14850 suggests that these two PKSs are expressed differently, although we have no evidence supporting this possibility. The lack of apparent PKS gene duplication events in the other three species could be due to repeat-induced point mutation (RIP). RIP occurs during sexual development and has been described in Fusarium graminearum and involves the introduction of nucleotide mutations into both copies of duplicated DNA (Cuomo et al. 2007). F. oxysporum does not have a known sexual phase and thus is unlikely to be exposed to RIP.

\section{Non-functional R-PKS domains}

Examination of each domain from each PKS, by BLAST analysis and AA alignments, indicate that all domains present in the 11
NR-PKSs are functional. In contrast, $16 \mathrm{ME}$ domains and $11 \mathrm{ER}$ domains in the 47 R-PKSs are likely to be non-functional. Alignment of all ME domains, spanning approximately 120 AAs, identified missing AAs and AA differences within one, two, three or all four of the AA motifs critical for ME function (Miller et al. 2003; Ansari et al. 2008) in the 16 PKSs. Figs 5A and $B$ are representative alignments of the putative functional and non-functional ME domains from the Fusarium verticillioides NR-PKSs. The functional ME domains contain four well-conserved motifs (Fig 5A) while comparable regions in the putative non-functional ME domains are very poorly conserved (Fig 5B) (Miller et al. 2003; Ansari et al. 2008). The dramatic differences in AA sequence emphasize the conclusion drawn from the phylogenetic analysis that the loss-of-function occurred on multiple occasions over time. BLASTp analysis of each ME domain provided additional evidence supporting whether or not the domains are functional. The functional ME domains shared significant deduced AA identity (average $E$-value $=1 \times 10^{-17}$ ) to the conserved ME domain, pfam0842, described in the National Center for Biotechnology Information (NCBI) Conserved Domain Database (CDD) (MarchlerBauer et al. 2005). The putative non-functional or remnant ME domains had no or very limited similarity to pfam08242 (average $E$-value $=4 \times 10^{-4}$ ). It is important to note that the structure of the four chemical products of the Fusarium NR-PKSs match the predicted functionality of their ME domain. The chemical structures of fusaric acid and zearalenone do not include any candidate ME-derived methyl groups, and the ME domains in the fusaric acid and zearalenone R-PKSs are likely non-functional based on the presence of poorly conserved ME motifs. In contrast, the chemical structures of fumonisins and fusarins include methyl groups (Fig 1), and the ME domains in the R-PKSs required for their production are predicted to be functional based on the presence of well-conserved ME motifs. A comparison of the putative non-functional ME domain of the fusaric acid PKS (FVEG_12523) with the functional domain of the fumonisin PKS (FVEG_00316) indicate that the two regions lack any sequence identity, which suggests that the functions of these regions are not the same.
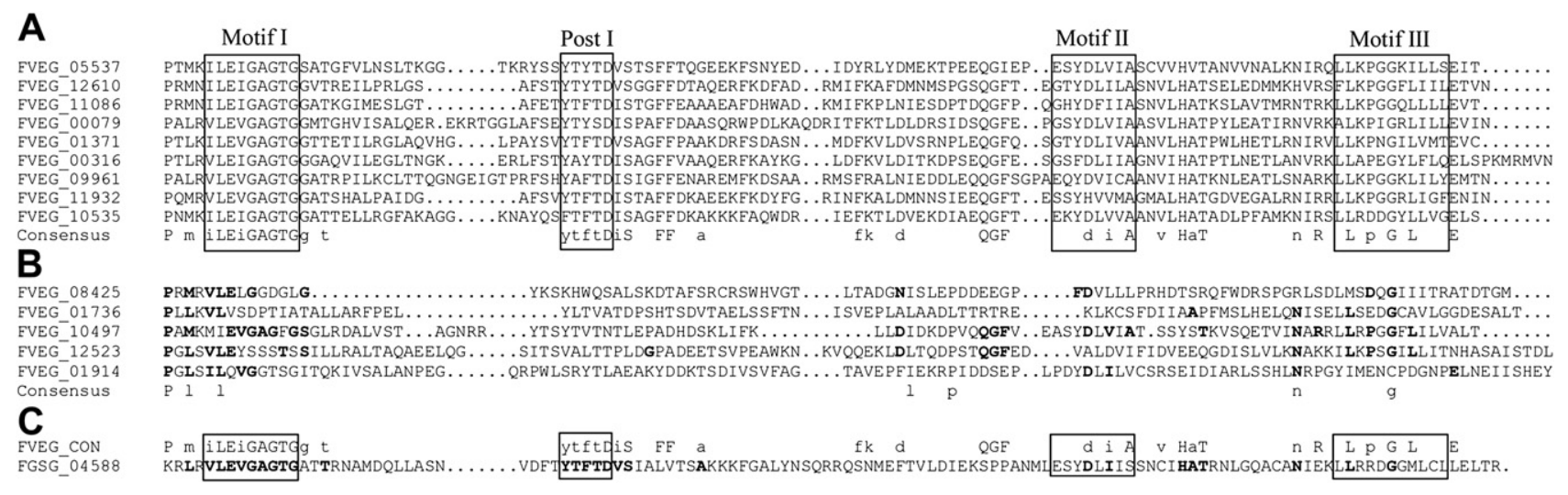

Fig 5 - Alignment of ME domains from F. verticillioides R-PKSs. (A) Putative functional ME domains. The four motifs previously identified in small molecule ME sequences are enclosed in boxes. (B) Putative non-functional ME domains. Conserved AAs are in bold text. (C) FVEG_CON list the conserved AA residues where a capital letter indicates complete conservation and a small case letter indicates significant conservation. FGSG_04588 indicates the AAs corresponding to the ME domain present at the carboxy-terminal end of the F. graminearum R-PKS FVEG_04588. Conserved AAs are in bold text. 
Alignment of all putative ER domains, which span approximately 230 AAs, also identified numerous gaps and differences in AA sequences among PKSs. Fig 6A depicts the AAs critical to ER function associated with binding reduced nicotinamide adenine dinucleotide phosphate (NADPH) (Song et al. 2004). This domain is well-conserved in 36 putative functional ER domains (Fig 6B) and poorly conserved in 11 putative non-functional ER domains (Fig 6C). BLASTp analysis of the putative functional ER domains indicated that they are significant similar (average $E$-value $=1 \times 10^{-45}$ ) to the conserved ER domain, cd05195, described in the NCBI CDD. In contrast, the putative non-functional ER domains were markedly less similar (average $E$-value $=4 \times 10^{-4}$ ) to cd05195. The loss of ER function is also reflected in the percent identity shared between non-functional and functional domains. The putative non-functional ER domain of the fusarin PKS (FVEG_11086) shares $24.6 \%$ identity with the functional ER domain of the fumonisin PKS (FVEG_00316), whereas the fumonisin PKS ER domain shares $45.2 \%$ identity with the functional ER domain of the fusaric acid PKS (FVEG_12523). The functionality of an ER domain cannot be predicted from a visual inspection of the polyketide product (Fig 1). Although the structure of lovastatin would suggest an ER is involved, the NR-PKS ER domain was found to be non-functional (Kennedy et al. 1999). The ER activity involved in lovastatin synthesis is coded for by a different gene located adjacent to the PKS (Kennedy et al. 1999). In contrast, the structure of fusarins suggests that their synthesis does not require ER activity, and this is reflected in the lack of a functional ER domain in the fusarin PKS according to the current analysis. These results are consistent with the findings of Song et al. 2004.

The distribution of non-functional ME and ER domains among Fusarium R-PKSs is significantly different. Non-functional ME domains are present in all three major clades of R-PKSs (Clades I-III), while non-functional ER domains occur only in R-PKS Clade II. In the case of the non-functional ME domains, their varied location within the tree and the pattern of AA insertions and deletions (indels) suggest that the initial DNA mutation leading to loss of ME function occurred multiple times during the evolution of Fusarium R-PKSs. JGI_98127, FGSG_08795, FVEG_01914, and FOXG_03051 are closely related

$$
\begin{aligned}
& \text { A General conserved ER domain: } \\
& \text { B R-PKS functional ER domains: } \\
& \text { C R-PKS nonfunctional ER domains: } \\
& \text { LIH } \times \times \times G G \times G \\
& \text { SVLIHXAXGGXGXAA }
\end{aligned}
$$

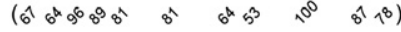

$$
\begin{aligned}
& \text {-VLIHXAX_ X XGXA } \\
& \left(93^{6} \hat{\imath}^{10} 8^{2} \text { is }^{3} 93^{6} b^{5} 0\right)
\end{aligned}
$$

Fig 6 - Critical motif for ER function. (A) Conserved nicotinamide adenine dinucleotide phosphate (NADPH) binding site of functionally characterized ER domains. Degree of conservation is indicated by the relative height of each letter and the percent identity shared by all 36 putative functional domains is indicated underneath each letter. (B) Putative functional NADPH binding domain and two flanking AAs from 36 Fusarium PKSs. (C) Putative non-functional NADPH binding domain in 11 Fusarium PKSs. An ' $x$ ' denotes a position where no AA is conserved greater than $50 \%$. orthologous R-PKSs that occur in the four species examined here and have putative non-functional ME domains. In the case of the PKSs with non-functional ER domains, all are located in a single clade (Clade II) suggesting that the loss-offunction may have been due to a single event, tracing back to a single PKS progenitor.

\section{Variability in NR-PKS domain organization}

The organization of the first five domains (SAT, KS, MAT, PT, and ACP) is the same in all 11 Fusarium NR-PKSs, but the type and number of domains near the carboxy-terminal region varies (Fig 3). For example, five of the NR-PKSs include an additional ACP domain; four of these are the PKS orthologues involved in synthesis of fusarubin. An additional ACP domain does not appear to affect product synthesis as site directed mutagenesis of a dual-ACP-containing PKS in the fungus Aspergillus revealed that each ACP domain was functional and able to operate independently of the other (Fujii et al. 2001). Here, the tree generated by phylogenetic analysis of Fusarium ACP domains from both dual and single-ACP PKSs was similar in topology with the tree generated with KS-AT/MAT from NR-PKSs (data not shown). In addition, the eight ACP domains from the four fusarubin PKS orthologues form two clades; the more carboxy-terminal ACPs form one clade (with $99 \%$ bootstrap support) and the more amino-terminal ACPs form the second clade (with $69 \%$ bootstrap support). The positions of NR-PKSs relative to each other in the ACP-based tree were the same as in the KS-AT-based tree (Fig 3). Results from a previous phylogenomic analysis of PKSs from diverse fungal genera (Kroken et al. 2003; Baker et al. 2006) suggest that the dual-ACP NRPKSs that currently exist in Fusarium and Aspergillus species did not arise independently in the two genera but instead are descended from a common ancestor.

The second difference near the carboxy terminus is the last domain. The C-terminal domain in all functionally characterized NR-PKSs is involved in determining chain length, final product cyclization and product release (Bailey et al. 2007; Du \& Lou 2010). We found that the terminal domains for the 11 Fusarium NR-PKSs to be either a TE (6), an R (4); or an ME (1). TE and R domains are the most commonly described PKS terminal domains and their role in product release have been extensively studied (Du \& Lou 2010). TE domains belong to the $\alpha / \beta$-hydrolase superfamily and may catalyze the release of a linear product with a terminal carboxylic acid or a cyclized product (Du \& Lou 2010). In contrast, R domains may catalyze release of a linear product with a terminal aldehyde or alcohol functionality via reduction of the acyl thioester, or the release of a product with one or more aromatic rings via a non-redox condensation reaction (Du \& Lou 2010). Thus, the presence of either a TE or $\mathrm{R}$ terminal domain can provide information about the chemical structure of polyketide synthesized by an NR-PKS. BLASTp analysis of the terminal domain of FGSG_04588 indicated that it shares significant identity $\left(E=4.49 \times 10^{-17}\right)$ with the conserved ME domain (pfam08242) described in the NCBI CDD database. In addition to similar BLASTp scores, all four ME motifs are well-conserved (Fig 5C). Although PksCT, involved in citrinin synthesis in Monascus purpureus has a similar type ME domain, it also 
includes a terminal TE domain (Shimizu et al. 2005). To our knowledge, no PKS with a C-terminal ME domain has been described in the literature and how or whether this terminal ME domain is involved in product release is not known.

\section{Acquisition of C-terminal domains}

Acquisition of an NRPS module and a putative carnitine/choline AT (CAT) domain at the carboxy-terminal end of R-PKSs appear to have occurred only once each, because NRPS domains occur only in R-PKS Clade II and CAT domains occur only in R-PKS Clade I (Fig 3). The NRPS module is a collection of four domains that span approximately 1400 AAs. The organization and putative functions of the four domains are completely conserved within all NRPS modules of the Fusarium R-PKSs. The four domains are responsible for condensation of the polyketide chain and an amino acid and then release of the resulting hybrid polyketide-amine molecule. Three PKSs (FGSG_10464, FVEG_10497, and FOXG_01189) within Clade II (the PKS-NRPS clade) lack an NRPS module. The positions of these three PKSs within Clade II suggest that the absence of the NRPS module can be explained by loss on at least two occasions: once in the branch leading to FGSG_10464 and once in the branch leading to FVEG_10497 and FOXG_01189. Loss of the NRPS module from FGSG_10464 appears to have occurred after divergence from two pairs of PKS-NRPSs in Fusarium verticillioides and Fusarium oxysporum (81 \% bootstrap support) (Fig 3). In contrast, at what point the NRPS module was lost in the branch leading to FVEG_10497 and FOXG_01189 is less clear because of the poor bootstrap support for branches leading to the well supported FVEG_10497/FOXG_01189 branch. A previous analysis also indicates that NRPS modules have been lost repeatedly from fungal R-PKSs (Kroken et al. 2003).

CAT enzymes are generally involved in the transport of acetyl-CoA across mitochondria and peroxisome membranes (van der Leij et al. 2000; Roze et al. 2011). They function by covalently attaching carnitine (L-3-hydroxy-4-aminobutyrobetaine) to the carboxylic acid portion of acetyl-CoA. A carnitine acylcarnitine translocase then moves the carnitine complex across a membrane after which another CAT reforms the two starting molecules (van Roermund et al. 1999; Roze et al. 2011). Aspergillus nidulans facC encodes a cytoplasmic CAT required for acetate utilization and acuJ encodes a mitochondrial and peroxisomal CAT required for fatty acid utilization (Hynes et al. 2011). BLAST analysis of the four Fusarium genomic sequences with $\mathrm{facC}$ and $a c u J$ as query sequences identified two CAT encoding genes in each genome which we designated CFT1 and CFT2 for carnitine (fatty acid) acyltransferase. The deduced AA sequences of these genes are $>90 \%$ identical to each other and $68 \%$ identical to FacC and AcuJ respectively (Hynes et al. 2011). The deduced AA sequences of CFT1 and CFT2 from the same species share $~ 25 \%$ identity.

R-PKS Clade I includes three PKSs with a terminal CAT domain (PKS-CAT): JGI_78513 and JGI_37058 from Fusarium solani and FGSG_05794 from Fusarium graminearum. Each CAT domain is $\sim 600$ AAs and has a high level of identity to AcuJ by BLASTp analysis (183-243 bits, $E=9 \times 10^{-48}$ to $2 \times 10^{-69}$ ) and to the conserved choline/carnitine $O$-acyltransferase, pfam00755, ( $E=1 \times 10^{-63}$ to $2 \times 10^{-69}$ ) described in the CDD. FOXG_10805 is likely a forth PKS-CAT in F. oxysporum, based on phylogenetic data (100\% bootstrap support) but, the nucleotide sequence coding for the CAT domain is missing from the genomic sequence data. A search of sequenced fungal genomes (at NCBI and JGI) identified 14 additional putative PKS-CATs from ten other fungi. Fungi containing this PKS group are present in five different classes from across the Ascomycota. It is interesting to note that most (83\%) of the fungi with PKS-CATs are pathogens of either plants (7), humans (1) or other fungi (2). Phylogenetic analysis of the 17 CAT domains from the PKS-CATs, CFT1 and CFT2 from all four fusaria, FacC and AcuJ from A. nidulans, two CATs from Candida glabrata, and one CAT from Saccharomyces cerevisiae, with a human CAT (EAW87874) serving as an outgroup, indicate that the CAT domain from fungal PKSs form a monophyletic group (100\% bootstrap support) (Fig 7). Our analysis also suggests that the ancestral PKS-CAT acquired the CAT domain from a cytoplasmic FacC-like CAT after the latter diverged from the mitochondrial AcuJ-like CAT.

To our knowledge, no polyketide synthesized by a PKS-CAT domain has been described in the literature. Although the function of the CAT domain likely involves the attachment of carnitine or carnitine-like moiety to the polyketide backbone generated by the PKS, the role of the moiety can only be speculated. One possibility is that the presence of the moiety enables the transport of the PKS product into a vesicle via a translocase. This possible mechanism to localize a secondary metabolite within an organelle is different than the mechanism described for synthesis of aflatoxin, where biosynthetic proteins are transported to vesicles designated aflatoxisomes (Roze et al. 2011). If PKS-CATs do indeed function to attach a carnitine to the nascent polyketide, it will interesting to determine if they are also capable of removing the carnitine, a function that both CFT1 and CFT2 confer.

\section{Transcriptional analysis}

Expression of the 16 Fusarium verticillioides PKSs was examined by analysis of EST and microarray data. Analysis of ESTs in the F. verticillioides Gene Index revealed transcripts for ten of the 16 PKSs in seven of ten cDNA libraries (Fig 8). Previously, we reported the expression of nine PKSs in a single microarray experiment (Ma et al. 2010). The corresponding secondary metabolites for five of the 16 PKSs are known. Here, we found evidence for the expression of all $16 \mathrm{~F}$. verticillioides PKSs in nine additional microarray studies (Fig 8). Although the pattern of expression of each gene is distinct, in some cases, the results have directed further studies. For example, the only PKS found to be expressed in live, symptomatic maize kernels during an ear rot infection assay was FVEG_13715. Preliminary studies found that FVEG_13715 insertional mutants are not affected in their ability to cause maize ear rot (Proctor, unpublished observations). Taken together, the expression data of the 16 PKSs is consistent with their being functional and involved in synthesis of polyketide product(s). A previous study also provided evidence for expression of 14 of 15 Fusarium graminearum PKSs (Gaffoor et al. 2005).

\section{Predicting PKS function}

Based on results from the phylogenetic analysis, coupled with previous published reports describing Fusarium metabolites, we predict the function of two Fusarium solani PKSs and 
three Fusarium oxysporum PKSs (Fig 3). Two PKSs, F. solani JGI_101778 and F. oxysporum FOXG_05816, belong to a group of four orthologous PKSs where the function of the other two has been determined; Fusarium verticillioides FVEG_03695 and
Fusarium graminearum FGSG_09182 are involved in the synthesis of the violet perithecial pigment (Gaffoor et al. 2005; Proctor et al. 2007). The recent discovery that FVEG_03695 is also required for fusarubin synthesis (Busman and Proctor,

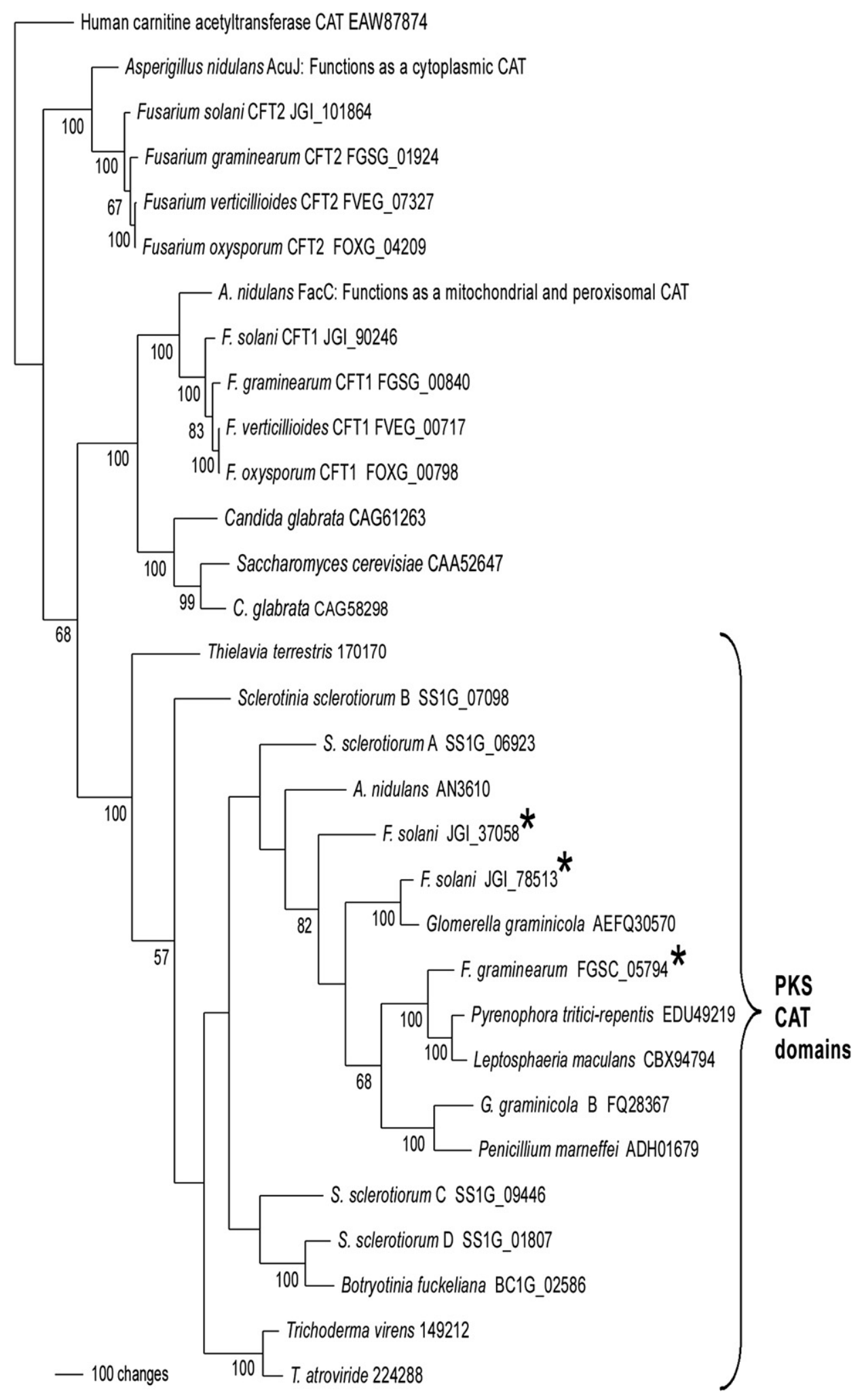

Fig 7 - Genealogy inferred by maximum parsimony analysis of deduced AA sequences of CAT domains from 17 filamentous fungal PKS-CATs and 13 CATs from Fusarium, Aspergillus nidulans, Candida glabrata, and Saccharomyces cerevisiae. The Fusarium PKS-CAT domains are indicated by an asterisk. 


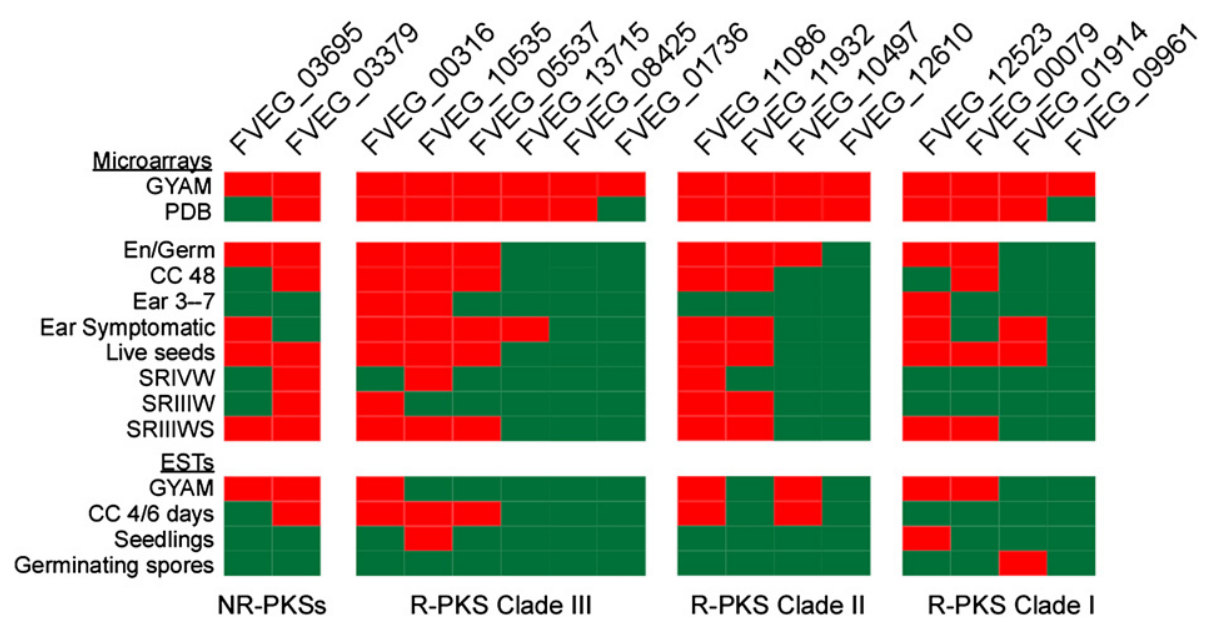

Fig 8 - Representation of change in expression observed for 16 F. verticillioides PKS genes by microarray and ESTs during growth on a variety substrates. The PKSs are labeled with the gene model name assigned at the Broad Fusarium Comparative Genomic Database. The PKSs are grouped according to phylogenetic analysis and are labeled at the bottom of the figure. Red represents relative increase in expression, green represents relative decrease in expression. See text for description of the microarray experiments and EST libraries.

personal communication) coupled with the observation that $F$. solani may produce fusarubin (Ammar et al. 1979) suggest that JGI_101778 is involved in fusarubin synthesis. The third PKS, F. oxysporum FOXG_04757 is part of a pair of orthologues of which its partner, F. verticillioides FVEG_03379 is likely involved in bikaverin synthesis (Brown et al. 2008; Brown et al. submitted for publication). FVEG_03379 shares $94.4 \%$ identity with the well characterized Fusarium fujikuroi bikaverin PKS (Wiemann et al. 2009). Coupled with the observation that F. oxysporum is reported to synthesize bikaverin (McInnes et al. 1976; Bell et al. 2003), we predict that FOXG_04757 is involved in bikaverin synthesis in F. oxysporum. Likewise, the fourth PKS, F. oxysporum FOXG_15248 is a closely related orthologue of F. verticillioides FVEG_11086, which is required for production of fusaric acid (Brown et al. submitted for publication). This, coupled with the observation that F. oxysporum is reported to produce fusaric acid (Bacon et al. 1996), we predict that FOXG_15248 is required for fusaric acid synthesis in F. oxysporum. The fifth PKS, F. solani JGI_70660 is a closely related orthologue of F. verticillioides FVEG_11086 and F. graminearum FGSG_07798, which are required for fusarin production in these fungi (Gaffoor et al. 2005; Brown et al. submitted for publication). The conservation of sequences flanking FVEG_11086, FGSG_07798, and JGI_101778 in the respective fungi (Gaffoor et al. 2005; Brown et al. submitted for publication) further supports the hypothesis thatJGI_101778 is involved in synthesis of fusarins or a structurally related metabolite(s) by F. solani. Demonstration of the predicted functions of these PKSs will require gene deletion and chemical analysis of the resulting mutants. The significant variability in polyketide structures observed, even for PKSs within the same clade, do not allow any further predictions to be made with confidence.

In a previous analysis of PKSs from a wide range of genera of the Ascomycota, the diversity and distribution of PKSs was attributed to gene duplication, divergence and differential gene loss rather than horizontal gene transfer (HGT) (Kroken et al. 2003). In contrast, the current analysis of Fusarium PKSs suggests that HGT could be responsible for significant PKS diversity in these fungi. If duplication was a major contributor to PKS diversity in Fusarium, one could expect to observe multiple examples of paralogous PKS genes in the Fusarium species examined. However, we observed evidence for only one pair of paralogous PKSs, FOXG_14850 and FOXG_15886 in F. oxysporum. In addition, of the $15 \mathrm{~F}$. graminearum PKSs and $16 \mathrm{~F}$. verticillioides PKSs, only five are closely related orthologues, and most of the rest are more closely related to PKSs from other fungal genera than they are to one another (Kroken et al. 2003; Baker et al. 2006). This indicates that most F. graminearum and $F$. verticillioides PKS genes began diverging from one another before Fusarium began diverging from other fungal genera. BLAST analyses also provide evidence that some F. oxysporum and F. solani PKSs are also more closely related to PKSs from other fungal genera than they are to other Fusarium PKSs. If divergence within Fusarium was a major contributor to PKS diversity in these fungi, we would expect that most Fusarium PKSs to be more similar to other Fusarium PKSs than they are to those in other fungi. However, this is not the case for most PKSs analyzed to date. HGT provides an explanation for the observed relationships of PKS from Fusarium and other fungi. An alternative explanation is that most Fusarium PKSs were present in the ancestral fusarium. If this were the case, the ancestral Fusarium would have had $~ 34$ different PKSs corresponding to the 34 groups of PKSs presented in Fig 4. However, these species represent only a portion of the genetic diversity within Fusarium and, therefore, probably only a portion of the PKS diversity in these fungi. Thus, the ancestral Fusarium would have had to have $>34$ PKSs. Given that the Fusarium species examined have only 12-16 PKSs, it seems unlikely that the genome of their common ancestor included at least 34 PKS genes. Although it is a complicated explanation for the diversity of PKSs in Fusarium, HGT is consistent with existing phylogenetic data on PKSs and does not require that 
the common ancestor was fundamentally different from extant species with respect to the number of PKS genes in its genome. The potential of HGT contributing to diversity of PKS genes in Fusarium is also consistent with two other observations: (1) multiple examples of HGT of SM biosynthetic gene clusters that include PKS gene between fungi (Khaldi \& Wolfe 2011; Slot \& Rokas 2011); and (2) the genomes of F. oxysporum and F. solani include a significant percentage of DNA that is not closely related to DNA in other Fusarium species but instead is more closely related to DNA in more distantly related organisms (Coleman et al. 2009; Ma et al. 2010). The later observation indicates that Fusarium includes species that have the ability to acquire DNA from other organisms, and this in turn could have facilitated HGT of PKS genes into Fusarium from other fungi.

Functional characterization of fungal PKSs is challenging and requires significant resources. Despite the considerable increase in F. graminearum research efforts since the release of its genome sequence (Trail 2009), the function of only one F. graminearum PKS in synthesis of a polyketide has been demonstrated since 2005 (Sørensen et al. 2011). We have performed a phylogenetic and domain structure analysis of PKSs encoded by four Fusarium genomes with the purpose of laying a foundation on which to design future experiments to elucidate the biological functions of polyketides produced by these fungi.

\section{Acknowledgements}

We would like to thank Chris McGovern, Crystal Probyn, and Marcie Moore for technical assistance. Mention of trade names or commercial products in this article is solely for the purpose of providing specific information and does not imply recommendation or endorsement by the US Department of Agriculture. USDA is an equal opportunity provider and employer.

\section{R E F E R E N C E S}

Altschul SF, Gish W, Miller W, Myers EW, Lipman DJ, 1990. Basic local alignment search tool. Journal of Molecular Biology 215: 403-410.

Ammar MS, Gerber NN, McDaniel L, 1979. New antibiotic pigments related to fusarubin from Fusarium solani. Journal of Antibiotics 32: 679-684.

Ansari MZ, Sharma J, Gokhale RS, Mohanty D, 2008. In silico analysis of methyltransferase domains involved in biosynthesis of secondary metabolites. BMC Bioinformatics 9: 1-21.

Bacon CW, Porter JK, Norred WP, Leslie JF, 1996. Production of fusaric acid by Fusarium species. Applied and Environmental Microbiology 62: 4039-4043.

Bailey AM, Cox RJ, Harley K, Lazarus CM, Simpson TJ, Skellam E, 2007. Characterisation of 3-methylorcinaldehyde synthase (MOS) in Acremonium strictum: first observation of a reductive release mechanism during polyketide biosynthesis. Chemical Communications 4053-4055.

Baker SE, Kroken S, Inderbitzin P, Asvarak T, Li BY, Shi L, Yoder OC, Turgeon BG, 2006. Two polyketide synthase-encoding genes are required for biosynthesis of the polyketide virulence factor,
T-toxin, by Cochliobolus heterostrophus. Molecular Plant-Microbe Interactions 19: 139-149.

Bell AA, Wheeler MH, Liu J, Stipanovic RD, Puckhaber LS, Orta H, 2003. United States Department of Agriculture-Agricultural Research Service studies on polyketide toxins of Fusarium oxysporum f sp vasinfectum: potential targets for disease control. Pest Management Science 59: 736-747.

Bennett JW, Bentley R, 1989. What's in a name?- microbial secondary metabolism. Advances in Applied Microbiology 34: $1-28$.

Bolstad BM, Irizarry RA, Astrand M, Speed TP, 2003. A comparison of normalization methods for high density oligonucleotide array data based on variance and bias. Bioinformatics 19: 185-193.

Brown DW, 2011. The KP4 killer protein gene family. Current Genetics 57: 51-62.

Brown DW, Butchko RA, Proctor RH, 2008. Genomic analysis of Fusarium verticillioides. Food Additives and Contaminants 25: 1158-1165.

Brown, Butchko, Busman, Proctor RH. Identification of gene clusters associated with fusaric acid, fusarin, and perithecial pigment production in Fusarium verticillioides. Fungal Genetics and Biology, submitted for publication.

Brown DW, Cheung F, Proctor RH, Butchko RA, Zheng L, Lee Y, Utterback T, Smith S, Feldblyum T, Glenn AE, Plattner RD, Kendra DF, Town CD, Whitelaw CA, 2005. Comparative analysis of 87,000 expressed sequence tags from the fumonisinproducing fungus Fusarium verticillioides. Fungal Genetics and Biology 42: 848-861.

Cole RJ, Jarvis BB, Schweikert MA, 2003. Handbook of Secondary Fungal Metabolites. Academic Press, San Diego.

Coleman JJ, Rounsley SD, Rodriguez-Carres M, Kuo A, Wasmann CC, Grimwood J, Schmutz J, Taga M, White GJ, Zhou S, Schwartz DC, Freitag M, Ma LJ, Danchin EG, Henrissat B, Coutinho PM, Nelson DR, Straney D, Napoli CA, Barker BM, Gribskov M, Rep M, Kroken S, Molnar I, Rensing C, Kennell JC, Zamora J, Farman ML, Selker EU, Salamov A, Shapiro H, Pangilinan J, Lindquist E, Lamers C, Grigoriev IV, Geiser DM, Covert SF, Temporini E, Vanetten HD, 2009. The genome of Nectria haematococca: contribution of supernumerary chromosomes to gene expansion. PLoS Genetics 5: 1-14.

Crawford JM, Townsend CA, 2010. New insights into the formation of fungal aromatic polyketides. Nature Reviews Microbiology 8: 879-889.

Cuomo CA, Guldener U, Xu JR, Trail F, Turgeon BG, Di Pietro A, Walton JD, Ma LJ, Baker SE, Rep M, Adam G, Antoniw J, Baldwin T, Calvo S, Chang YL, Decaprio D, Gale LR, Gnerre S, Goswami RS, Hammond-Kosack K, Harris LJ, Hilburn K, Kennell JC, Kroken S, Magnuson JK, Mannhaupt G, Mauceli E, Mewes HW, Mitterbauer R, Muehlbauer G, Munsterkotter M, Nelson D, O’Donnell K, Ouellet T, Qi W, Quesneville H, Roncero MI, Seong KY, Tetko IV, Urban M, Waalwijk C, Ward TJ, Yao J, Birren BW, Kistler HC, 2007. The Fusarium graminearum genome reveals a link between localized polymorphism and pathogen specialization. Science 317: 1400-1402.

Desjardins AE, Plattner RD, Proctor RH, 1996. Linkage among genes responsible for fumonisin biosynthesis in Gibberella fujikuroi mating population A. Applied and Environmental Microbiology 62: 2571-2576.

Desjardins AE, Proctor RH, 2007. Molecular biology of Fusarium mycotoxins. International Journal of Food Microbiology 119: 47-50.

Devereux J, Haeberli P, Smithies O, 1984. A comprehensive set of sequence analysis programs for the VAX. Nucleic Acids Research 12: $387-395$.

Du L, Lou L, 2010. PKS and NRPS release mechanisms. Natural Product Reports 27: 255-278. 
Fujii I, Watanabe A, Sankawa U, Ebizuka Y, 2001. Identification of Claisen cyclase domain in fungal polyketide synthase WA, a naphthopyrone synthase of Aspergillus nidulans. Chemistry \& Biology 8: 189-197.

Gaffoor I, Brown DW, Plattner R, Proctor RH, Qi W, Trail F, 2005. Functional analysis of the polyketide synthase genes in the filamentous fungus Gibberella zeae (anamorph Fusarium graminearum). Eukaryotic Cell 4: 1926-1933.

Glenn AE, 2007. Mycotoxigenic Fusarium species in animal feed. Animal Feed Science and Technology 137: 213-240.

Graziani S, Vasnier C, Daboussi MJ, 2004. Novel polyketide synthase from Nectria haematococca. Applied and Environmental Microbiology 70: 2984-2988.

Hopwood DA, Khosla C, 1992. Genes for polyketide secondary metabolic pathways in microorganisms and plants. In: Chadwick DJ, Whelan J (eds), Secondary Metabolites: their function and evolution. John Wiley and Sons, Chichester, UK, pp. 88-112.

Hynes MJ, Murray SL, Andrianopoulos A, Davis MA, 2011. Role of carnitine acetyltransferases in acetyl coenzyme A metabolism in Aspergillus nidulans. Eukaryotic Cell 10: 547-555.

Irizarry RA, Hobbs B, Collin F, Beazer-Barclay YD, Antonellis KJ, Scherf U, Speed TP, 2003. Exploration, normalization, and summaries of high density oligonucleotide array probe level data. Biostatistics 4: 249-264.

Keller NP, Turner G, Bennett JW, 2005. Fungal secondary metabolism - from biochemistry to genomics. Nature Reviews Microbiology 3: 937-947.

Kennedy J, Auclair K, Kendrew SG, Park C, Vederas JC, Hutchinson CR, 1999. Modulation of polyketide synthase activity by accessory proteins during lovastatin biosynthesis. Science 284: 1368-1372.

Khaldi N, Wolfe KH, 2011. Evolutionary origins of the fumonisin secondary metabolite gene cluster in Fusarium verticillioides and Aspergillus niger. International Journal of Evolutionary Biology 2011: 1-7.

Kroken S, Glass NL, Taylor JW, Yoder OC, Turgeon BG, 2003. Phylogenomic analysis of type I polyketide synthase genes in pathogenic and saprobic ascomycetes. Proceedings of the National Academy of Sciences 100: 15670-15675.

Ma LJ, van der Does HC, Borkovich KA, Coleman JJ, Daboussi MJ, Di Pietro A, Dufresne M, Freitag M, Grabherr M, Henrissat B, Houterman PM, Kang S, Shim WB, Woloshuk C, Xie X, Xu JR, Antoniw J, Baker SE, Bluhm BH, Breakspear A, Brown DW, Butchko RA, Chapman S, Coulson R, Coutinho PM, Danchin EG, Diener A, Gale LR, Gardiner DM, Goff S, Hammond-Kosack KE, Hilburn K, Hua-Van A, Jonkers W, Kazan K, Kodira CD, Koehrsen M, Kumar L, Lee YH, Li L, Manners JM, Miranda-Saavedra D, Mukherjee M, Park G, Park J, Park SY, Proctor RH, Regev A, Ruiz-Roldan MC, Sain D, Sakthikumar S, Sykes S, Schwartz DC, Turgeon BG, Wapinski I, Yoder O, Young S, Zeng Q, Zhou S, Galagan J, Cuomo CA, Kistler HC, Rep M, 2010. Comparative genomics reveals mobile pathogenicity chromosomes in Fusarium oxysporum. Nature 464: 367-373.

Marasas WF, Riley RT, Hendricks KA, Stevens VL, Sadler TW, Gelineau-Van Waes J, Missmer SA, Cabrera J, Torres O, Gelderblom WC, Allegood J, Martinez C, Maddox J, Miller JD, Starr L, Sullards MC, Roman AV, Voss KA, Wang E, Merrill Jr AH, 2004. Fumonisins disrupt sphingolipid metabolism, folate transport, and neural tube development in embryo culture and in vivo: a potential risk factor for human neural tube defects among populations consuming fumonisincontaminated maize. Journal of Nutrition 134: 711-716.

Marchler-Bauer A, Anderson JB, Cherukuri PF, DeWeese-Scott C, Geer LY, Gwadz M, He S, Hurwitz DI, Jackson JD, Ke Z, Lanczycki CJ, Liebert CA, Liu C, Lu F, Marchler GH, Mullokandov M, Shoemaker BA, Simonyan V, Song JS,
Thiessen PA, Yamashita RA, Yin JJ, Zhang D, Bryant SH, 2005. CDD: a Conserved Domain Database for protein classification. Nucleic Acids Research 33: D192-D196.

McInnes AG, Walter JA, Smith DG, Wright JL, Vining LC, 1976. Biosynthesis of bikaverin in Fusarium oxysporum. Use of ${ }^{13} \mathrm{C}$ nuclear magnetic resonance with homonuclear ${ }^{13} \mathrm{C}$ decoupling to locate adjacent ${ }^{13} \mathrm{C}$ labels. Journal of Antibiotics 29: 1050-1057.

Miller DJ, Ouellette N, Evdokimova E, Savchenko A, Edwards A, Anderson WF, 2003. Crystal complexes of a predicted S-adenosylmethionine-dependent methyltransferase reveal a typical AdoMet binding domain and a substrate recognition domain. Protein Science 12: 1432-1442.

Morgavi DP, Riley RT, 2007. An historical overview of field disease outbreaks known or suspected to be caused by consumption of feeds contaminated with Fusarium toxins. Animal Feed Science and Technology 137: 201-212.

O'Donnell K, Sutton DA, Fothergill A, McCarthy D, Rinaldi MG, Brandt ME, Zhang N, Geiser DM, 2008. Molecular phylogenetic diversity, multilocus haplotype nomenclature, and in vitro antifungal resistance within the Fusarium solani species complex. Journal of Clinical Microbiology 46: 2477-2490.

Proctor RH, Butchko RA, Brown DW, Moretti A, 2007. Functional characterization, sequence comparisons and distribution of a polyketide synthase gene required for perithecial pigmentation in some Fusarium species. Food Additives and Contaminants 24: 1076-1087.

Proctor RH, Desjardins AE, Plattner RD, Hohn TM, 1999. A polyketide synthase gene required for biosynthesis of fumonisin mycotoxins in Gibberella fujikuroi mating population A. Fungal Genetics and Biology 27: 100-112.

Roze LV, Chanda A, Linz JE, 2011. Compartmentalization and molecular traffic in secondary metabolism: a new understanding of established cellular processes. Fungal Genetics and Biology 48: 35-48.

Seefelder W, Humpf HU, Schwerdt G, Freudinger R, Gekle M, 2003. Induction of apoptosis in cultured human proximal tubule cells by fumonisins and fumonisin metabolites. Toxicology and Applied Pharmacology 192: 146-153.

Shim WB, Flaherty JE, Woloshuk CP, 2003. Comparison of fumonisin $B_{1}$ biosynthesis in maize germ and degermed kernels by Fusarium verticillioides. Journal of Food Protection 66: 2116-2122.

Shimizu T, Kinoshita H, Ishihara S, Sakai K, Nagai S, Nihira T, 2005. Polyketide synthase gene responsible for citrinin biosynthesis in Monascus purpureus. Applied and Environmental Microbiology 71: 3453-3457.

Slot JC, Rokas A, 2011. Horizontal transfer of a large and highly toxic secondary metabolic gene cluster between fungi. Current Biology 21: 134-139.

Song Z, Cox RJ, Lazarus CM, Simpson TJ, 2004. Fusarin C biosynthesis in Fusarium moniliforme and Fusarium venenatum. ChemBioChem 5: 1196-1203.

Sørensen JL, Hansen FT, Sondergaard TE, Wimmer R, Droce A, Giese H, Frandsen RJN, 2011. Targeted activation of the polyketide synthase 9 gene cluster in Fusarium graminearum. In: 26th Fungal Genetics Conference at Asilomar, (Suppl.), p. 55.

Sydenham EW, Thiel PG, Marasas WFO, Shephard GS, Schalkwyk DJ, Koch KR, 1990. Natural occurrence of some Fusarium mycotoxins in corn from low and high esophageal cancer prevalence areas of the Transkei, Southern Africa. Journal of Agricultural and Food Chemistry 38: 1900-1903.

Trail F, 2009. For blighted waves of grain: Fusarium graminearum in the postgenomics era. Plant Physiology 149: 103-110.

van der Leij FR, Huijkman NC, Boomsma C, Kuipers JR, Bartelds B, 2000. Genomics of the human carnitine acyltransferase genes. Molecular Genetics and Metabolism 71: 139-153.

van Roermund CW, Hettema EH, van den Berg M, Tabak HF, Wanders RJ, 1999. Molecular characterization of carnitinedependent transport of acetyl-CoA from peroxisomes to 
mitochondria in Saccharomyces cerevisiae and identification of a plasma membrane carnitine transporter, Agp2 p. ЕMBO Journal 18: 5843-5852.

Voss KA, Smith GW, Haschek WM, 2007. Fumonisins: toxicokinetics, mechanism of action and toxicity. Animal Feed Science and Technology 137: 299-325.
Wiemann P, Willmann A, Straeten M, Kleigrewe K, Beyer M, Humpf HU, Tudzynski B, 2009. Biosynthesis of the red pigment bikaverin in Fusarium fujikuroi: genes, their function and regulation. Molecular Microbiology 72: 931-946.

Wu F, 2007. Measuring the economic impacts of Fusarium toxins in animal feeds. Animal Feed Science and Technology 137: 363-374. 